\title{
ASPECTOS BÁSICOS SOBRE LA ECOLOGÍA DE LA NUTRIA DE RÍO (Lontra longicaudis annectens) PARA LA COSTA DE OAXACA
}

\author{
MA. ANTONIETA CASARIEGO-MADORELL, RURIK LIST \\ Y GERARDOCEBALLOS \\ Instituto de Ecología, UNAM. Apartado Postal 70-275, México, D.F. 04510 \\ madorell@prodigy.net.mx
}

\begin{abstract}
Southern river otter densities of 0.03 to $0.62 / \mathrm{km}$ of river were estimated for three rivers of the coast of the Mexican State of Oaxaca by the number of feces found on transects. The estimated population for the 19 perennial rivers of the state was 495 . Scat analysis showed that crustaceans, fish, insects and amphibians are the main components of the otter's diet.
\end{abstract}

Keywords: Lontra longicaudis, density, abundance, diet, Ayuta, Copalita, Zimatán.

Palabras clave: Lontra longicaudis, densidad, abundancia, alimentación, Ayuta, Copalita, Zimatán.

La nutria de río (Lontra longicaudis annectens) habita en casi todos los ríos, presas, lagos y lagunas costeras de América Latina. Son abundantes en los ríos donde la vegetación ribereña es densa y las raíces de los árboles forman galerías. Los ríos con este tipo de vegetación usualmente son de aguas claras, flanqueados por grandes bloques rocosos (Parera, 1996; Lariviére, 1999). En México la nutria presenta una distribución amplia y continua, abarcando desde el sur de Tamaulipas por el Golfo y hasta el norte de Chihuahua y Sonora por el Pacífico (Aranda, 2000). Se alimentan principalmente de peces, crustáceos y pequeños mamíferos, aunque también puede alimentarse de algunas aves, anfibios, reptiles y ocasionalmente de insectos y frutos (Gallo, 1989; Quadros y Monteiro, 2000; Soler-Frost, 2004). En la legislación mexicana, se encuentra en la categoría de amenazada de acuerdo a la NOM-059-ECOL-2002. Sus poblaciones han sido seriamente diezmadas en algunas zonas, por factores como la cacería, destrucción de su hábitat y contaminación de las aguas (Gallo, 1986; Alho et al., 1988; Chehéber, 1991).

En el estado de Oaxaca se sabe poco o casi nada sobre su biología. En esta región se realizó un estudio en julio de 1999 a agosto del 2000 en los ríos Ayuta, Copalita y Zimatán en la costa de Oaxaca entre los $15^{\circ} 43^{\prime}$ y $16^{\circ} 50^{\prime}$ N y $95^{\circ} 45^{\prime}$ y $96^{\circ} 15^{\prime}$ W. Este polígono es parte de un área prioritaria para la conservación (RTP-129). Comprende una superficie de 60,000 ha dentro de los municipios de San Pedro Huamelula, Santiago Astata y San Miguel del Puerto, con corrientes permanentes con 
varias corrientes intermitentes como el río Chacalapa y lagunas costeras como Mezcalco. El clima es cálido subhúmedo (Aw) con una temperatura media anual de $28^{\circ}$ C, una precipitación promedio anual de 700 a $800 \mathrm{~mm}$. Se caracteriza por presentar una estacionalidad con dos temporadas bien definidas 1) la época de lluvias de mayo a octubre y 2) la época de secas de noviembre a abril (INEGI, 1988). Los principales tipos de vegetación son el bosque tropical caducifolio y la selva tropical subcaducifolia a la orilla de los ríos; hacia la porción norte en los sitios cercanos a las serranías se encuentran selvas tropicales subperenifolias y en los alrededores de las lagunas costeras angostas franjas de manglar (Rzedowski, 1978; García et al., 1992).

En este estudio se estimó el tamaño poblacional y alimentación de la nutria en los tres ríos por medio de 3 transectos de $2 \mathrm{~km}$ para cada río. Se contaron y colectaron los excrementos para determinar el tipo de alimentación. Utilizando el modelo de Gallo (1986) y el de Eberhardt y Van Etten modificado (Casariego-Madorell, 2004). Se calcularon $0.03 \pm 0.1$ nutrias $/ \mathrm{km}$ lineal en el río Ayuta, que no es muy profundo, $\mathrm{y}$ durante el muestreo en la temporada seca segmentos del río se secaron en su totalidad. Para el río Copalita, se calcularon $0.26 \pm 0.06$ nutrias $/ \mathrm{km}$ lineal, este río presenta paredes de roca de granito de hasta $3 \mathrm{~m}$ de altura y zonas de arena donde se pudieron encontrar sitios para formar sus madrigueras como lo mencionan Pardini y Trajano (1999). Sin embargo, durante la época de lluvia, la corriente lleva mucho sedimento (Salas-Morales, 2002).

Para el río Zimatán se calcularon $0.62 \pm 0.15$ nutrias $/ \mathrm{km}$ lineal. En este río se encuentran madrigueras de nutria en el segundo transecto entre las $16^{\circ} 53^{\prime \prime} \mathrm{N} \mathrm{y} 96^{\circ} 00^{\prime \prime}$ W. Este río presenta áreas con una amplia cobertura de vegetación, con varias pozas, agua clara y está poco perturbado (Casariego-Madorell, 2004; Salas-Morales, 2002).

Con el promedio de las estimaciones para los ríos Copalita y Zimatán, utilizando ambos modelos, se obtiene una abundancia promedio de $0.42 \pm 0.07$ nutrias/ $\mathrm{km}$ lineal, se estima una población de 495 nutrias aproximadamente para los 19 ríos permanentes que conforman la costa de Oaxaca. No se tomó en cuenta el río Ayuta para obtener el tamaño poblacional debido a que la mayoría de las veces fue imposible el acceso a los transectos, por lo que para disminuir el sesgo únicamente se consideraron dos ríos. Los resultados obtenidos sugieren que la población de la costa es aparentemente viable, por lo que es necesario establecer un programa de monitoreo, en éstos y otros ríos a lo largo de las costa, para dar un seguimiento a la población y poder detectar de forma oportuna amenazas a la población de nutrias y/o la salud de los ríos.

En el análisis de las excretas se determinó una alimentación compuesta principalmente de crustáceos (53.0\%), peces (33.1\%), insectos (9.8\%) y anfibios (4.0 $\%$ ), destacando los crustáceos de la familia Palaemonidae (Macrobrachium americanum y M. acanthochirus), y los peces destaca la familia Gobiesocidae (Gobiexos mexicanus) el cual es una especie endémica y bajo protección especial de acuerdo a la NOM-059-ECOL-2002. 
Las nutrias son especies muy sensibles a la perturbación y contaminación, por lo que su permanencia depende del estado de conservación de los cuerpos de agua, la reducción de las poblaciones de nutrias o su desaparición es una señal de alteración a las condiciones de los sistemas acuáticos, por lo que los estudios sobre esta especie proveen información oportuna para la conservación de estos sistemas.

\section{AGRADECIMIENTOS}

Agradeciendo el apoyo financiero y logístico del Fondo Mundial para la Conservación de la Naturaleza (WWF). Sociedad para el Estudios de los Recursos Bióticos de Oaxaca (SERBO). Denver Zoological Foundation. Idea Wild, Inc. Laboratorio de Ictiología del Instituto de Biología UNAM. Al Dr. Brian Miller por todo su apoyo, a Cuauhtemoc Chávez por su ayuda en las cuestiones estadísticas y a Yolanda Domínguez por los comentarios sugeridos al texto.

\section{LITERATURACITADA}

Alho, C.J.R., T.E. Lancher, JR., y H.C. Goncalves. 1988. Environmental degradation in the Pantanal ecosystem. Bioscience, 38:164-171.

Aranda, J.M. 2000. Huellas y otros rastros de los mamíferos grandes y medianos de México. $1^{a}$ ed. Instituto de Ecología A. C. Xalapa, Veracruz, México.

Casariego Madorell, M.A. 2004. Abundancia relativa y hábitos alimetnarios de la nutria de río (Lontra longicaudis annectens) en la costa de Oaxaca, México. Tesis de Maestria. Posgrado en Ciencias Biológicas. Facultad de Ciencias, UNAM. México.

Chehéber, C. 1991. Searching for the giant otter in northeastern Argentina. International Union for the Conservation of Nature, Otter Specialist Group Bulletin, 6:17-18.

Gallo, J.P. 1986. Otters in Mexico. Journal of the Otter Trust, 1:19-24.

Gallo, J.P. 1989. Distribución y estado actual de la nutria o perro de agua (Lutra longicaudis annectens Major, 1897) en la Sierra Madre del sur, México. Tesis de Maestría, Facultad de Ciencias UNAM.

García, G., S. Salas., L. Schibli., R. Aguilar., S. Acosta, y A. Salazar. 1992. Análasis de la vegetación y usos actuales del suelo en el estado de Oaxaca. Fase I (Costa y Sierra sur).Informe técnico SERBO. AC.

INEGI. 1988. Carta de efectos climáticos. Juchitán. ED15-10D-15-1. Instituto Nacional de Estadística. Geografía e Informática.

Lariviére, S. 1999. Lontra longicaudis. Mammalian Species, 609: 1-5.

Pardini, R. y E. Trajano. 1999. Use of shelters by the neotropical river otter (Lontra longicaudis) in an Atlantic forest stream, southeastern Brazil. Journal of Mammalogy, 80:600-610.

Parera, A. 1996. Estimating river otter Lutra longicaudis population in Iberá lagoon using a direct sightings methodology. IUCN Otter Specialist Group Bulletin, 13:77-83. 
Quadros, J. y Emygdio L.A. Monteiro-Filho. 2000. Fruit occurrence in the diet of the neotropical otter, Lontra longicaudis, in southern Brazilian Atlantic forest and its implication for seed dispersion. Journal Neotropical Mammal, 7:33-36.

Rzedowski, J. 1978. Vegetación de México. Ed. Limusa, México.

Salas-Morales, S.H. 2002. Relación entre la heterogeneidad ambiental y la variabilidad estructural de las selvas tropicales secas de la costa de Oaxaca, México. Tesis de Maestira. Facultad de Ciencias, UNAM. D.F.

SEMARNAT (Secretaría de Medio Ambiente y Recursos Naturale) 2002. Norma Oficial Mexicana NOM-059-ECOL-2001. Protección ambiental - especies nativas de México de flora y fauna silvestres - Categorías de riesgo y especificaciones para su inclusión, exclusión o cambio - Listas de especies en riesgo. Diario Oficial, 6 de marzo de 2002, 56 pp.

Soler-Frost, A.M. 2004. Cambios en la abundancia relativa y dieta de Lontra longicaudis en relación a la perturbación de la Selva Lacandona, Chiapas, México. Tesis de Licenciatura Facultad de Ciencias, UNAM. México. 